\title{
Microscale Patterning of Electrochromic Polymer Films via Soft Lithography
}

\author{
Jae-Yeong Jung, ${ }^{1}$ Seokwon Joo, ${ }^{2}$ Da-Seul Kim, ${ }^{1}$ Kyoung-Hwan Kim, ${ }^{1}$ Tae Soup Shim (D), ${ }^{1,3}$ \\ Soonmin Seo $\mathbb{D}^{2},{ }^{2}$ and Ju-Hyung Kim ${ }^{1,3}$
}

${ }^{1}$ Department of Energy Systems Research, Ajou University, Suwon 16499, Republic of Korea

${ }^{2}$ College of BioNano Technology, Gachon University, Gyeonggi 13120, Republic of Korea

${ }^{3}$ Department of Chemical Engineering, Ajou University, Suwon 16499, Republic of Korea

Correspondence should be addressed to Tae Soup Shim; tsshim@ajou.ac.kr, Soonmin Seo; soonmseo@gachon.ac.kr, and Ju-Hyung Kim; juhyungkim@ajou.ac.kr

Received 13 July 2018; Accepted 23 October 2018; Published 13 December 2018

Academic Editor: Miriam H. Rafailovich

Copyright (c) 2018 Jae-Yeong Jung et al. This is an open access article distributed under the Creative Commons Attribution License, which permits unrestricted use, distribution, and reproduction in any medium, provided the original work is properly cited.

\begin{abstract}
We present a direct fabrication technique of patterned polymeric electrochromic (EC) devices via soft lithography, enabling both negative patterning and positive patterning of the polymer. For this work, elastomeric polydimethylsiloxane (PDMS) molds were employed as not only stamps for direct contact printing of polymer inks but also templates for dewetting of polymer solutions under mild experimental conditions. We performed both negative patterning and positive patterning of a prototypical EC polymer and investigated the EC device characteristics according to solvents, solution concentrations, and pattern types. Eventually, the complex patterns, which cannot be realized by conventional shadow masking processes, and large-area structures were successfully demonstrated. We anticipate that these results will be applied to the development of various patterned devices and circuits, which may lead to further applications.
\end{abstract}

\section{Introduction}

In the past few decades, electrochromic (EC) materials and devices have attracted a lot of interest for the prospect of potential applications such as optical displays, optical switching devices, antiglare mirrors, and smart windows [1-5]. The EC materials reversibly change their optical properties (i.e., light transmittance, absorbance, and reflectance) in response to external bias voltages, resulting from electrochemical oxidation and reduction. Various classes of materials including transition metal oxides and conducting polymers have been widely studied as promising EC materials thus far [6-9]. In particular, considerable research efforts have been devoted to the EC behaviors of conducting polymers owing to their outstanding advantages of low cost, mechanical flexibility, and solution processability in comparison with inorganic materials [10-15].
For practical applications of EC devices, patterning processes of the EC layers in thin-film form are necessarily required in accordance with their intended uses [10-16]. However, conventional patterning processes such as photolithography and shadow mask deposition are mostly inappropriate for conducting polymers because the polymers are significantly damaged or even broken down under harsh processing conditions involved in etching or physical vapor deposition [17]. Solution processability of conducting polymers rather facilitates various soft lithographic patterning methods using elastomeric polydimethylsiloxane (PDMS) molds in a wide range of scales [17-20]. The PDMS molds can be employed as not only stamps for direct contact printing of polymer inks but also templates for dewetting of polymer solutions under mild experimental conditions, owing to the low modulus and surface energy of PDMS leading to conformal contact and easy release. Even though 
those methods enable both negative patterning and positive patterning of the polymers without any harmful damages, only a few attempts have been made to apply them to fabricating the patterned EC devices thus far.

Here, we demonstrate the fabrication of patterned polymeric EC devices via soft lithographic methods. For this work, poly(3-hexylthiophene-2,5-diyl) (P3HT) was used as a prototypical EC material in consideration of excellent EC properties (i.e., high coloration efficiency, high optical contrast, and rapid switching time), compatibility with solution processes, and functional versatility [13, 21-25]. We performed both negative patterning and positive patterning of the polymer using the PDMS molds consisting of pillar arrays and investigated the EC device characteristics according to solvents, solution concentrations, and pattern types. In addition, more complex patterns (such as island and ring arrays), which cannot be realized by conventional shadow masking processes, and large-area structures were successfully achieved. These results suggest great potential for the further development of various patterned EC devices and circuits, including display pixels, optical switching devices, and smart window applications.

\section{Materials and Methods}

2.1. Preparation of PDMS Molds. The PDMS molds used in this work were replicated from initially patterned photoresist layers. Spin-coated photoresist (SU-8 2025, MicroChem) layers (of $12 \mu \mathrm{m}$ thickness) were engraved with the hole patterns in accordance with the previous reports [26, 27]. The diameters of the hole motifs were designed to be 50 and $100 \mu \mathrm{m}$. The patterned photoresist layers were then negatively replicated using a PDMS elastomer (Sylgard 184, Dow Corning). A mixture of PDMS prepolymer and curing agent (in a 10:1 weight ratio) was poured onto each patterned photoresist layer and subsequently degassed and cured in a vacuum oven at $70^{\circ} \mathrm{C}$ for $3 \mathrm{hr}$. After thermal curing, the PDMS replicas were easily peeled off from the photoresist layers due to the low surface energy of PDMS, and consequently, the PDMS molds with the pillar arrays (of 50 and $100 \mu \mathrm{m}$ diameters) were prepared [28].

\subsection{Fabrication of Patterned Polymeric EC Devices. Patterned} polymeric EC devices using P3HT (Sigma-Aldrich) as an active component were fabricated on glass substrates coated with indium-tin-oxide (ITO) via both negative and positive patterning processes of the polymer. The ITO-coated glass substrates were cleaned before use by successive sonication in deionized water, ethyl alcohol, acetone, and isopropyl alcohol for $10 \mathrm{~min}$ each. P3HT was individually dissolved in chlorobenzene and chloroform with varied concentrations (i.e., $0.10,0.25,0.50,1.00,2.00$, and $3.00 \mathrm{wt} \%$ ) for solution processes, and all solutions were filtered through polyvinylidene fluoride (PVDF) membranes with $0.45 \mu \mathrm{m}$ pore size.

To prepare the negatively patterned P3HT layers (i.e., negative hole arrays of the PDMS pillars) on the ITO surfaces, the P3HT solutions were drop-dispensed onto the ITO-coated glass substrates, and then the PDMS molds were brought into contact with the samples as schematically illustrated in Figure 1. Since structural deformation of the PDMS molds can be induced by solvent swelling [29], an external pressure of 0.1 bar was uniformly applied during the process for ensuring conformal contact between PDMS and ITO. The PDMS structure with low surface energy leads to dewetting of the polymer solutions, accompanying solvent evaporation under ambient conditions, and thus, the negatively patterned P3HT layers are simultaneously formed on the ITO surfaces. In the case of the positive patterns of P3HT (i.e., same patterns as the PDMS pillar arrays), the PDMS molds were used as stamps for direct contact printing of the polymer, as shown in Figure 1. The P3HT solutions $(100 \mu \mathrm{L}$ each) were preferentially spin-coated at $1000 \mathrm{rpm}$ for $30 \mathrm{~s}$ onto the PDMS molds to form the patterned layers, and the solvents were subsequently removed in the vacuum oven at $80^{\circ} \mathrm{C}$ for $20 \mathrm{~min}$ to avoid solvent swelling effects [29]. For enhancing the adhesion properties between P3HT and ITO, both P3HT and ITO surfaces were treated using oxygen plasma for $1 \mathrm{~min}$ with a power of $10 \mathrm{~W}$ at a constant oxygen flow rate of 30 standard cubic centimeters per minute (sccm) [17], and then the patterned P3HT layers were directly transferred onto the ITO-coated glass substrates by applying an external pressure of 0.02 bar for $10 \mathrm{~s}$. Note that the patterned P3HT layers were also observed using scanning electron microscopy (SEM) (COXEM, EM-30AX) at an accelerating voltage of $20 \mathrm{kV}$ with a working distance of $\sim 8 \mathrm{~mm}$. For the SEM measurements, the samples were coated with gold (of $\sim 10 \mathrm{~nm}$ thickness) using a sputter coater.

To complete the EC device structure, each prepared sample was covered with another ITO-coated glass, and a gap distance between the sample and the cover was fixed using adhesive tapes (of $270 \mu \mathrm{m}$ thickness) as shown in Figure 1(b). The gap space was then filled with an electrolyte solution $\left(0.1 \mathrm{M}\right.$ of $\mathrm{LiClO}_{4}$ in propylene carbonate) by capillary action. The device characteristics were measured using a Keithley 2636 source/measure unit.

\section{Results and Discussion}

Figure 2(a) shows the negatively patterned P3HT layer using the PDMS mold with the pillar array of $100 \mu \mathrm{m}$ diameter, which was successfully achieved on the ITO-coated glass substrate with chloroform as a solvent. Since chloroform is rapidly evaporated and easily permeates into PDMS, the complete negative pattern (i.e., open-hole array) was distinctly formed at a high concentration of $3 \mathrm{wt} \%$. On the other hand, since the surface energy of P3HT $\left(\sim 36.0 \mathrm{~mJ} / \mathrm{m}^{2}\right)$ is significantly higher than that of the PDMS mold [17, 30-32], the P3HT-enriched solutions induced by solvent evaporation enhance the dewetting behaviors. Such dewetting behaviors of the P3HT-enriched solutions are also confirmed by the contact angle measurements. The contact angle of the P3HT solution on the PDMS surface gradually increases with increasing the solution concentration (see Supplementary Figure 1). Thus, accompanying with solvent evaporation, the less-viscous solutions with longer evaporation times (i.e., diluted solutions) are expelled from the molds before completing the formation of the patterns. These phenomena are intensified with diluting the solution, and accordingly, 


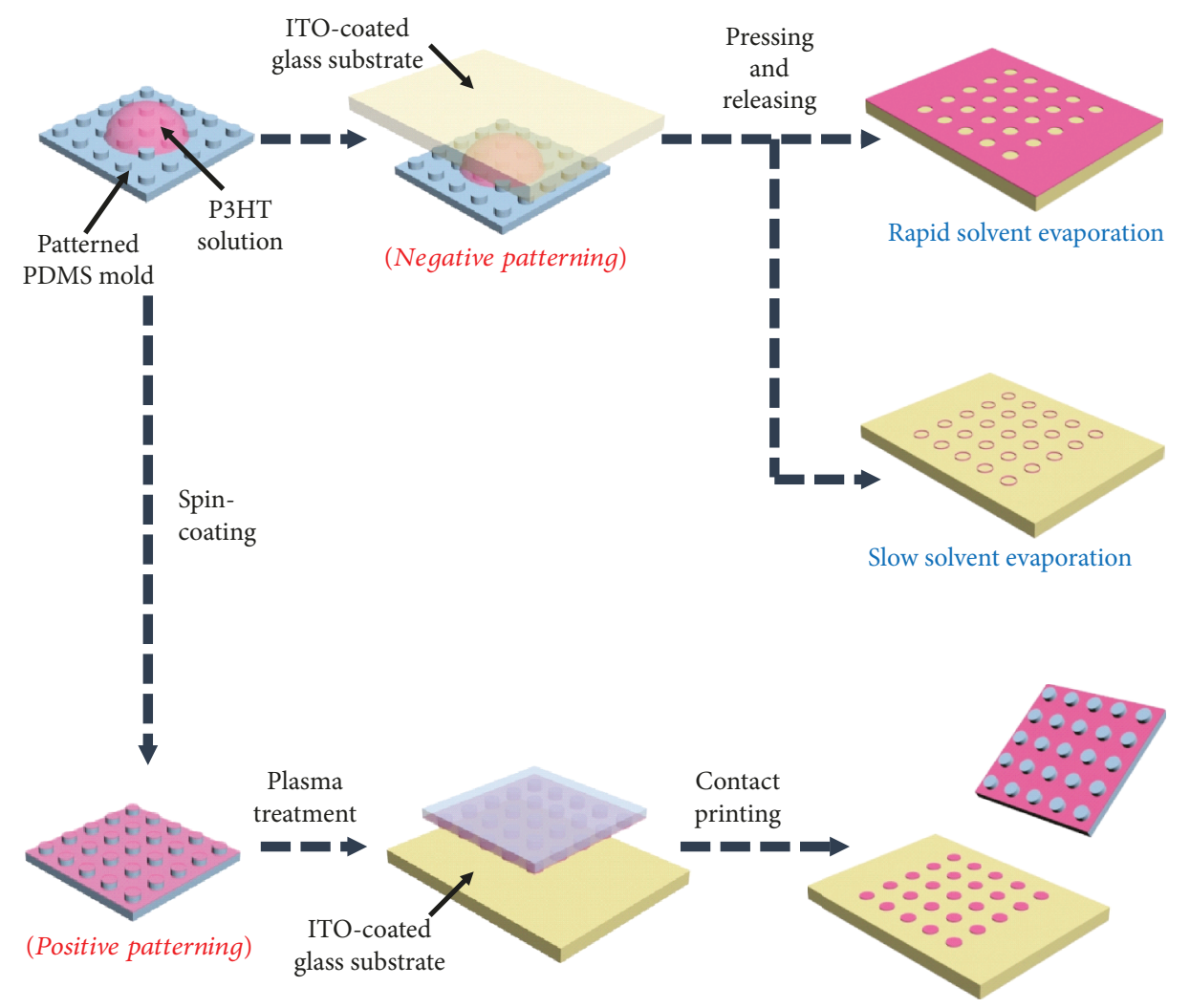

(a)

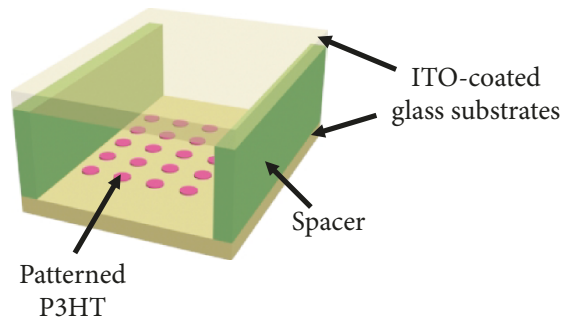

(b)

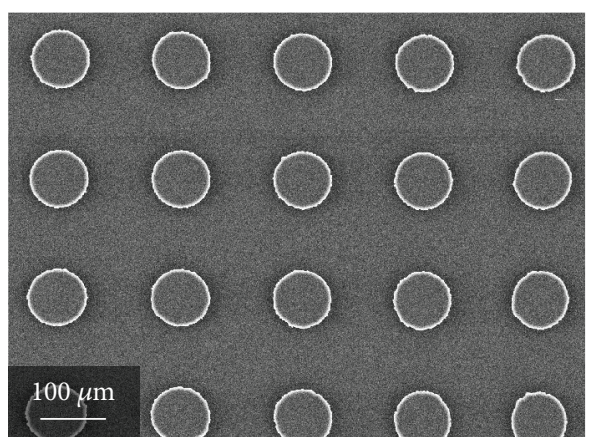

(c)

FIgURE 1: (a) Schematic illustration of the fabrication of patterned P3HT layers via both negative and positive patterning processes. (b) Configuration of EC display device. The gap space is filled with an electrolyte solution by capillary action. (c) SEM image of the PDMS mold with the pillar array of $100 \mu \mathrm{m}$ diameter.

smaller amounts of $\mathrm{P} 3 \mathrm{HT}$ are aggregated along the corners of the pillars due to the surface tension at the edges (see Figures 2 and 3) [33]. Note that the solution with a high concentration of $3 \mathrm{wt} \%$ using chloroform as a solvent was not seriously dewetted by solvent evaporation, which may originate from high viscosity and rapid evaporation of the solvent. These results imply that well-defined patterning of the polymer corresponding to the sharp outlines of the patterns can be performed if the residual layers of $\mathrm{P} 3 \mathrm{HT}$ are sufficiently removed. In this context, the formation of the residual layers is progressively suppressed with increasing the solvent evaporation time. For instance, in the case of chloroform, the solutions at low concentrations (less than $0.25 \mathrm{wt} \%$ ) rarely formed the residual layers as shown in
Figure 2(e). This tendency is more clearly observed using a less-volatile solvent such as chlorobenzene. Chlorobenzene exhibits lower volatility and less permeates into PDMS in comparison with chloroform [34], which can provide enough time to expel the solutions without remaining residues even at high concentrations. Figure 2 also shows the negatively patterned P3HT layers which were prepared with chlorobenzene as a solvent following the same procedure as for chloroform. Almost no residual layers were observed as the concentration increased up to $1 \mathrm{wt} \%$, and the well-defined patterns were distinctly formed along the outlines of the pillars (i.e., ring array). The fabrication of the complete negative pattern (i.e., open-hole array) was not achievable using chlorobenzene, and only the residual layers slightly 


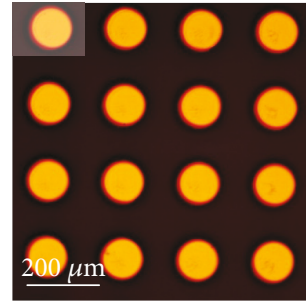

Chloroform 3.00 wt\%

(a)

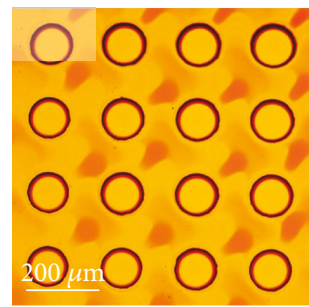

Chlorobenzene $2.00 \mathrm{wt} \%$

(f)

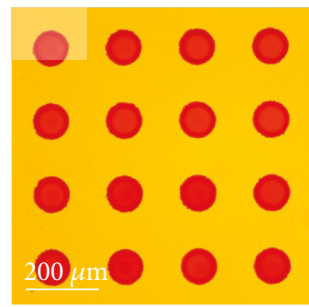

Chloroform $3.00 \mathrm{wt} \%$

(k)

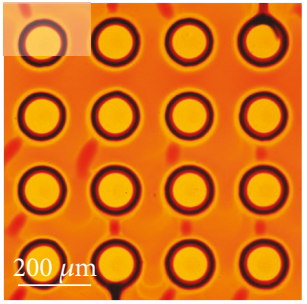

Chloroform 2.00 wt $\%$

(b)

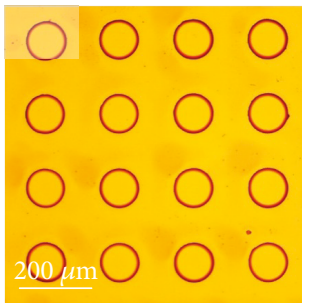

Chlorobenzene 1.00 wt $\%$

(g)

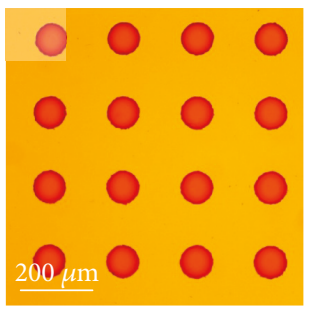

Chloroform $2.00 \mathrm{wt} \%$

(1)

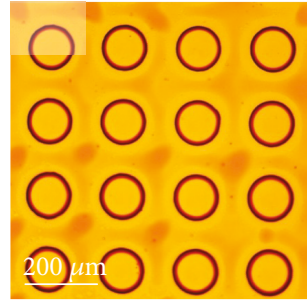

Chloroform 1.00 wt\%

(c)

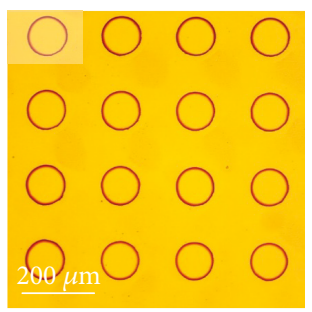

Chlorobenzene $0.50 \mathrm{wt} \%$

(h)

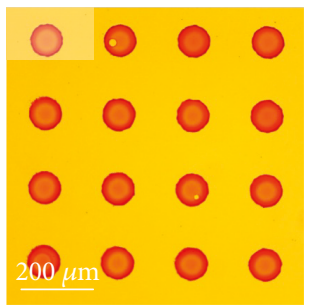

Chloroform 1.00 wt\%

(m)

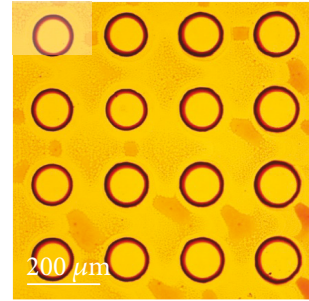

Chloroform 0.50 wt\%

(d)

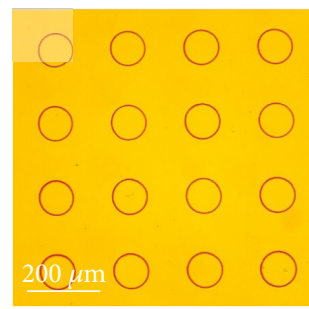

Chlorobenzene 0.25 wt $\%$

(i)

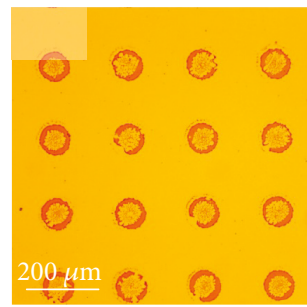

Chloroform $0.50 \mathrm{wt} \%$

(n)

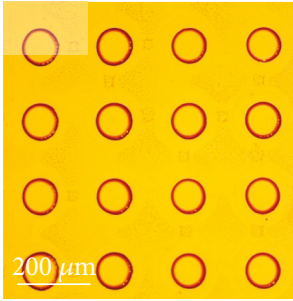

Chloroform 0.25 wt $\%$

(e)

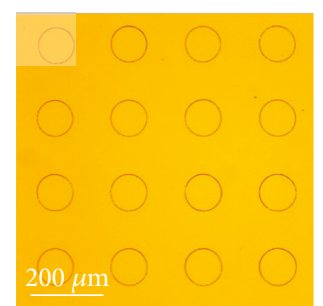

Chlorobenzene 0.10 wt\%

(j)

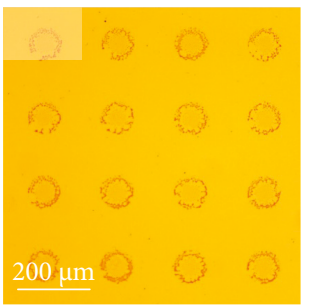

Chloroform 0.25 wt\%

(o)

Figure 2: Optical microscopy images of the patterned P3HT layers using the PDMS mold with the pillar array of $100 \mu \mathrm{m}$ diameter. (a-e) The negatively patterned P3HT layers using chloroform as a solvent. The PDMS molds were used as templates for dewetting of the polymer solutions, accompanying with solvent evaporation. The solution concentrations were (a) 3.00, (b) 2.00, (c) 1.00, (d) 0.50 , and (e) 0.25 wt\%, respectively. ( $\mathrm{f}-\mathrm{j})$ The negatively patterned P3HT layers, prepared with chlorobenzene as a solvent following the same procedure as for chloroform. The solution concentrations were (f) 2.00, (g) 1.00, (h) 0.50 , (i) 0.25 , and (j) $0.10 \mathrm{wt} \%$, respectively. (k-o) The positively patterned P3HT layers via direct contact printing. The chloroform solvent was solely used for spin-coating deposition of the polymer on the PDMS surface due to serious dewetting issues. The solution concentrations were (k) 3.00 , (l) 2.00 , (m) 1.00 , (n) 0.50 , and (o) $0.25 \mathrm{wt} \%$, respectively.

remained even at higher concentrations above $2 \mathrm{wt} \%$ as shown in Figure 2(f). It is worth noting that the overall tendency for the polymer patterning (i.e., surface tension and dewetting effects) was not significantly changed according to the size of the pillars in a micrometer scale (see Supplementary Figure 2). In particular, the contact angles of water droplets on both flat and patterned PDMS surfaces were measured to be within the range of $100^{\circ}$ to $110^{\circ}$ without any distinct difference, of which corresponding surface energies (from 16.8 to $22.7 \mathrm{~mJ} / \mathrm{m}^{2}$ ) are sufficiently lower than that of P3HT [17]. Figure 3 also shows an exemplary P3HT pattern fabricated using the PDMS mold with a smaller pillar array (of $\sim 1 \mu \mathrm{m}$ thickness). In addition, such negative and closed-outline patterns (i.e., hole and ring arrays) cannot be achieved by typical shadow masking methods, and thus, our demonstration shows promise as an easy process for realizing various complex patterns and their related applications.

The positively patterned P3HT layers were successfully fabricated on the ITO-coated glass substrates via direct contact printing using the same PDMS molds as for the negative patterning (see Figure 2 and Supplementary Figure 2). For this work, the P3HT solutions with varied concentrations were spin-coated on the PDMS molds to form the patterned layers and subsequently dried in a vacuum oven for avoiding solvent swelling effects [29]. Note that chlorobenzene was found to be not an appropriate solvent for spin-coating deposition of the polymer on the PDMS surface due to dewetting issues, and the complete deposition of P3HT could be achieved only using chloroform at an appropriate spin speed ( $1000 \mathrm{rpm})$. After oxygen plasma treatments for enhancing the adhesion properties [17], the deposited P3HT layers were brought into contact with the ITO-coated glass substrates and pressed (at 0.02 bar) to ensure the conformal contact. The patterned P3HT layers in the contact regions (i.e., filled circle array) were sharply transferred onto the substrates. Note that the edges of the transferred patterns were thicker than the other parts, because larger amounts of the polymer are piled up near 


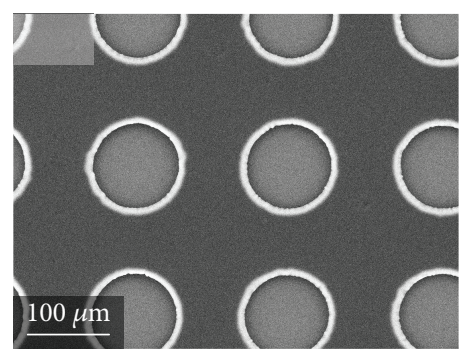

(a)

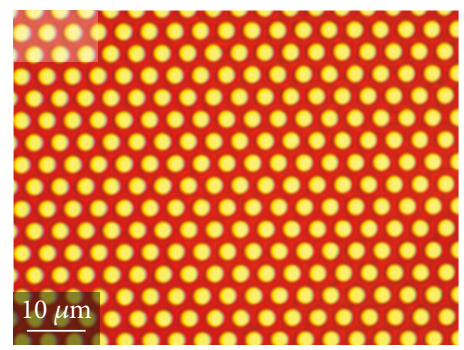

(c)

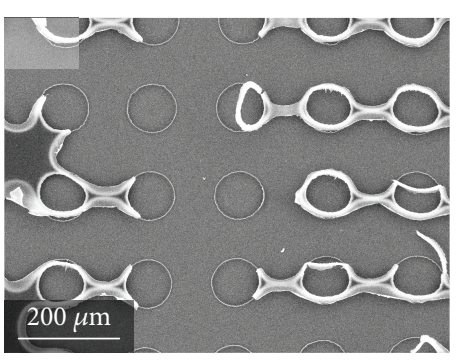

(b)

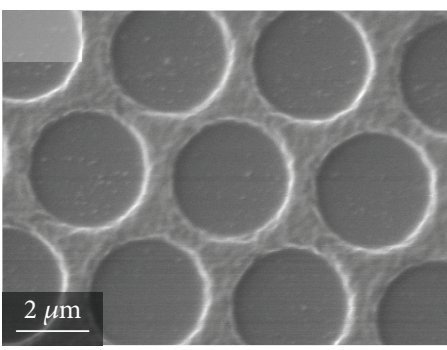

(d)

FIGURE 3: (a) SEM image of the complete negative pattern (i.e., open-hole array) of P3HT, distinctly formed at a high concentration of $3.00 \mathrm{wt} \%$ using chloroform as a solvent. (b) SEM image of the negatively patterned P3HT layer formed at a concentration of $2.00 \mathrm{wt} \%$ using chloroform as a solvent. The solution was expelled from the mold before completing the formation of the pattern, and accordingly, a small amount of P3HT was aggregated along the corners of the pillars in comparison with (a). (c) Optical microscopy image of an exemplary P3HT pattern fabricated using the PDMS mold with a smaller pillar array (of $\sim 1 \mu \mathrm{m}$ thickness). A significant change in the overall tendency for the polymer patterning was not observed according to the size of the pillars. (d) SEM image of (c).

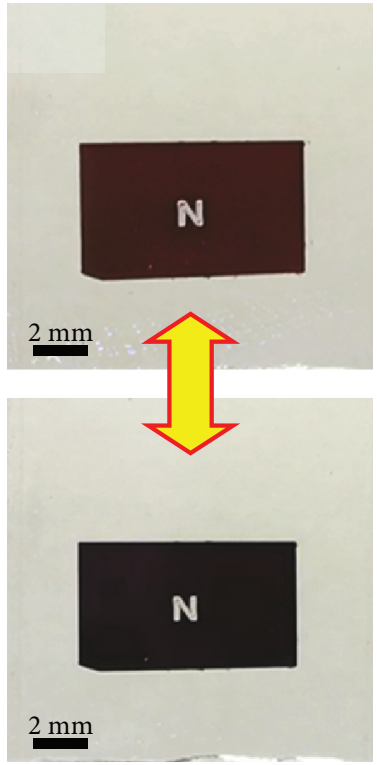

(a)

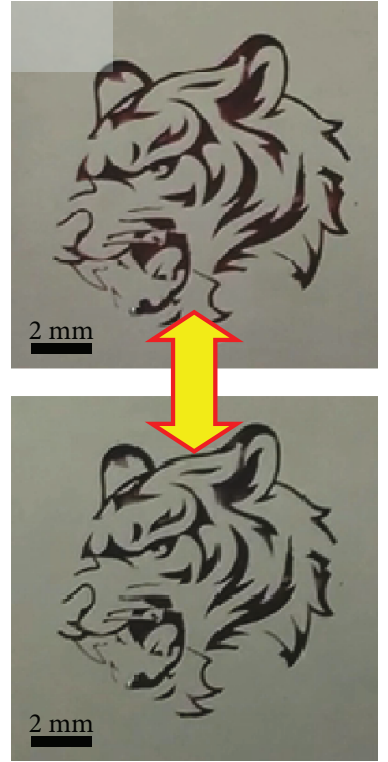

(b)

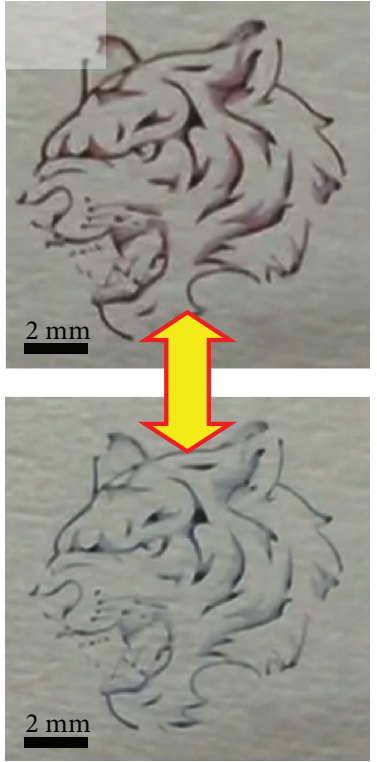

(c)

FIgUre 4: Photographs of the EC display devices with (a) negatively patterned P3HT layer at a concentration of $3.00 \mathrm{wt} \%$, (b) positively patterned P3HT layer at a concentration of $3.00 \mathrm{wt} \%$, and (c) positively patterned P3HT layers at a concentration of $1.00 \mathrm{wt} \%$. All devices were fabricated using chloroform as a solvent. Changes in colors of the P3HT layers were clearly observed according to the reversible oxidation (upper: red) and reduction (lower: blue) reactions in the electrolyte solution.

the edges during the spin-coating processes owing to the surface tension $[35,36]$. Such thickness deviation is mitigated as the overall thickness increases with increasing the solution concentration (see Figure 2(k)).
Figure 4 shows demonstrations of the polymeric EC display devices with large-area patterns $\left(\sim 1 \mathrm{~cm}^{2}\right)$, which were prepared by both negative and positive patterning processes. Changes in the color of the polymer were clearly 


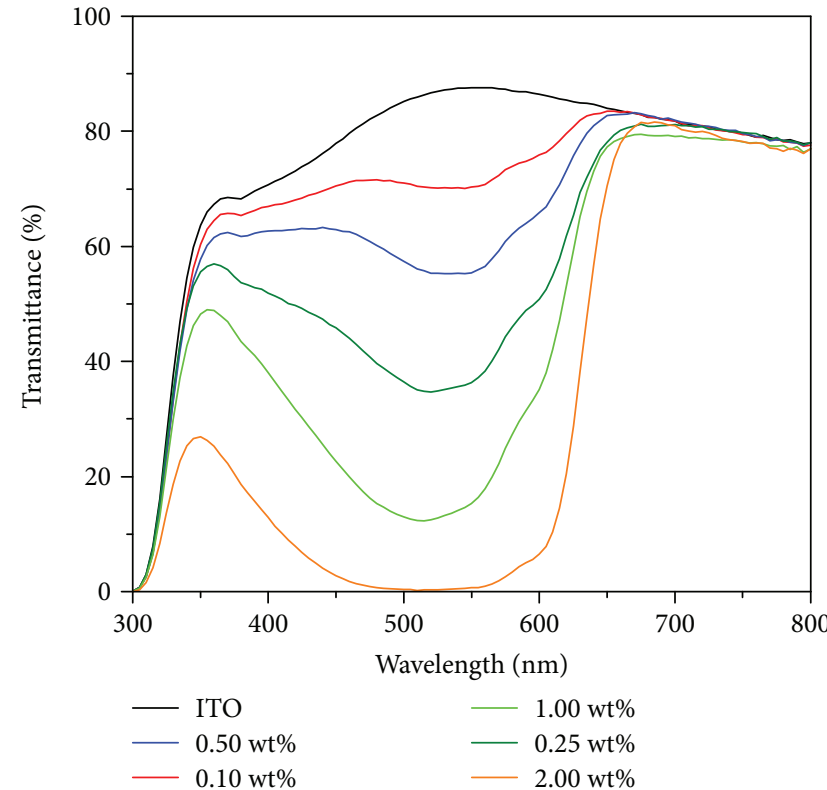

(a)

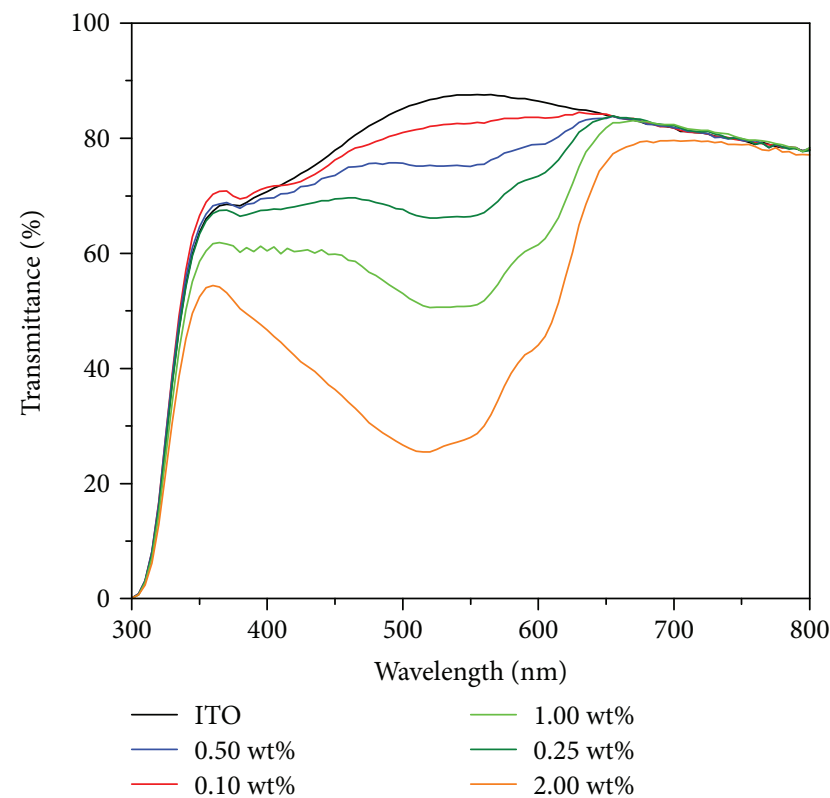

(c)

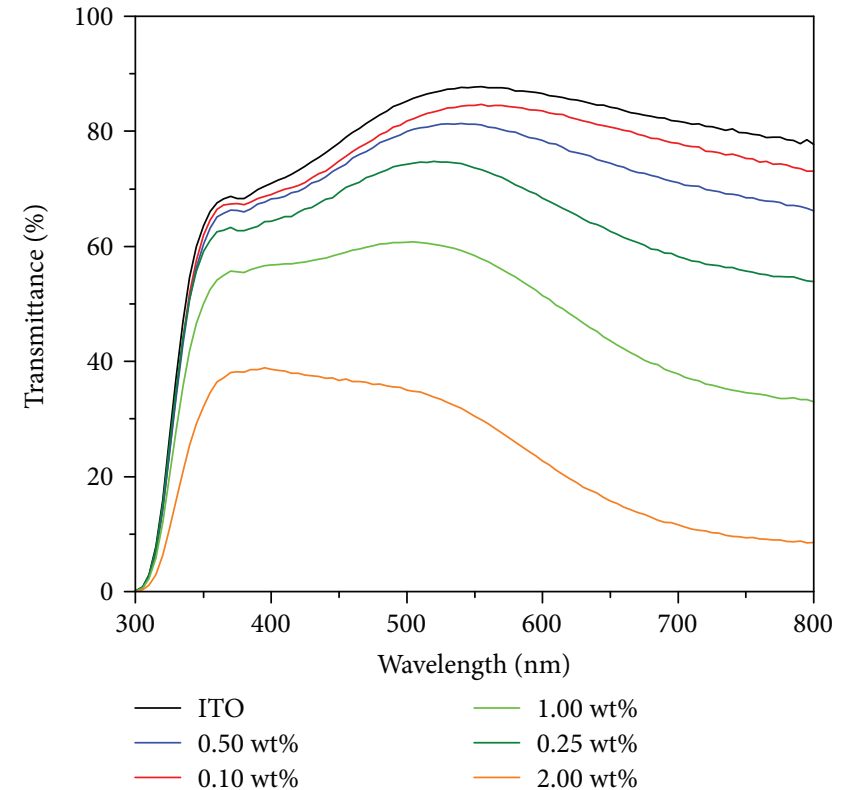

(b)

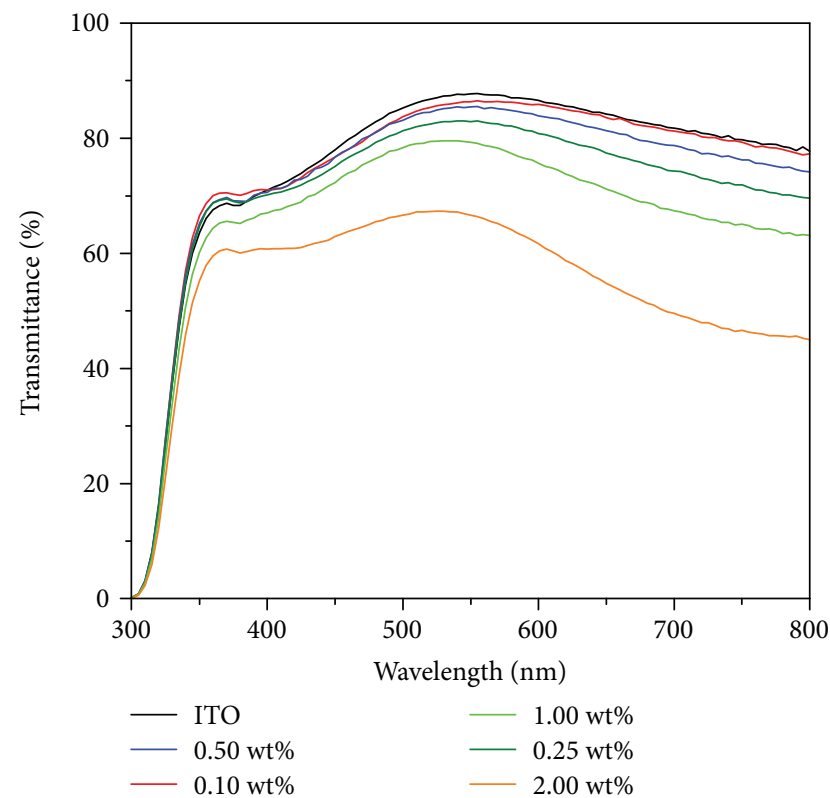

(d)

FIGURE 5: Light transmittances of the spin-coated P3HT films on the ITO substrates according to the solution concentrations, in the range of $300 \mathrm{~nm}$ to $800 \mathrm{~nm}$. The spin-coating processes were performed using (a, b) chloroform and (c, d) chlorobenzene as solvents, respectively. The spectra in (a) and (c) correspond to the P3HT oxidation (red) states, and the spectra in (b) and (d) correspond to the P3HT reduction (blue) states.

observed according to the reversible oxidation (red) and reduction (blue) reactions in the electrolyte solution (see Supplementary Movie 1). For the EC operations, the sample bias voltage between the two electrodes was kept at $-3.0 \mathrm{~V}$ for reduction and $+3.0 \mathrm{~V}$ for oxidation. As indicated in Figure 5, light transmittance of the P3HT film, leading to color contrast, significantly varies with the film thickness rather than the type of solvents [37]. The film thickness can be easily modified by the solution concentration, and the rapid solvent evaporation of chloroform enables the formation of the thicker films in comparison with chlorobenzene. For the $0.10,0.25,0.50,1.00$, and $2.00 \mathrm{wt} \%$ solutions using the chloroform (chlorobenzene) solvent, the film thicknesses were measured to be $<10 \mathrm{~nm}(<10 \mathrm{~nm})$, $13 \mathrm{~nm}(<10 \mathrm{~nm}), 30 \mathrm{~nm}(14 \mathrm{~nm}), 100 \mathrm{~nm}(25 \mathrm{~nm})$, and $320 \mathrm{~nm}(50 \mathrm{~nm})$, after spin-coating deposition onto the 
substrates. The EC response time of P3HT was measured to be less than a second.

\section{Conclusions}

In conclusion, we demonstrate the fabrication of patterned polymeric EC devices via soft lithographic methods, enabling both negative patterning and positive patterning of the polymer. The PDMS molds with pillar arrays were employed for the patterning of $\mathrm{P} 3 \mathrm{HT}$ as the prototypical EC polymer with the excellent EC properties (i.e., high coloration efficiency, high optical contrast, and rapid switching time). For the negative patterning, the PDMS molds were used as templates for dewetting of the polymer solutions, accompanying with solvent evaporation. The complete negative (i.e., hole arrays) and the distinct closed-outline (i.e., ring arrays) patterns were selectively achieved without significant residual defects according to the volatility and permeability of the solvent. For the positive patterning, the direct contact printing method using the PDMS molds as the stamps was successfully performed via suppressing the solvent swelling effects and enhancing the adhesion properties. Eventually, the large-area structures were successfully fabricated, and the EC device characteristics were also investigated according to solvents, solution concentrations, and pattern types. In addition, the complex patterns (such as island and ring arrays) presented in this work cannot be realized by typical shadow masking methods. Thus, it is anticipated that these results will significantly contribute not only to the fabrication of various polymer patterns but also to the development of more diversified EC devices and circuits leading to a wide range of applications.

\section{Data Availability}

The data used to support the findings of this study are available from the corresponding author upon request.

\section{Conflicts of Interest}

There are no conflicts to declare.

\section{Acknowledgments}

This work was supported by the Ajou University research fund.

\section{Supplementary Materials}

Supplementary 1. Supplementary Figure 1: contact angles of the $\mathrm{P} 3 \mathrm{HT}$ solutions with varied concentrations (i.e., $0.00,1.00,2.00$, and $3.00 \mathrm{wt} \%$ ) on the PDMS surface using chloroform and chlorobenzene as solvents. Supplementary Figure 2: optical microscopy images of the patterned P3HT layers using the PDMS mold with the pillar array of the $50 \mu \mathrm{m}$ diameter.

Supplementary 2. Supplementary Movie 1: demonstration of the polymeric EC display device with a large-area pattern $\left(\sim 1 \mathrm{~cm}^{2}\right)$.

\section{References}

[1] C. M. Lampert, "Electrochromic materials and devices for energy efficient windows," Solar Energy Materials, vol. 11, no. 1-2, pp. 1-27, 1984.

[2] R. D. Rauh, "Electrochromic windows: an overview," Electrochimica Acta, vol. 44, no. 18, pp. 3165-3176, 1999.

[3] E. S. Lee and D. L. DiBartolomeo, "Application issues for large-area electrochromic windows in commercial buildings," Solar Energy Materials \& Solar Cells, vol. 71, no. 4, pp. 465491, 2002.

[4] R. J. Mortimer, A. L. Dyer, and J. R. Reynolds, "Electrochromic organic and polymeric materials for display applications," Displays, vol. 27, no. 1, pp. 2-18, 2006.

[5] P. Andersson, R. Forchheimer, P. Tehrani, and M. Berggren, "Printable all-organic electrochromic active-matrix displays," Advanced Functional Materials, vol. 17, no. 16, pp. 30743082, 2007.

[6] R. J. Mortimer, "Electrochromic materials," Chemical Society Reviews, vol. 26, no. 3, pp. 147-156, 1997.

[7] P. R. Somani and S. Radhakrishnan, "Electrochromic materials and devices: present and future," Materials Chemistry and Physics, vol. 77, no. 1, pp. 117-133, 2003.

[8] S. K. Deb, "Opportunities and challenges in science and technology of WO3 for electrochromic and related applications," Solar Energy Materials \& Solar Cells, vol. 92, no. 2, pp. 245-258, 2008.

[9] P. M. Beaujuge and J. R. Reynolds, "Color control in $\pi$-conjugated organic polymers for use in electrochromic devices," Chemical Reviews, vol. 110, no. 1, pp. 268-320, 2010.

[10] D. Hohnholz, H. Okuzaki, and A. G. MacDiarmid, "Plastic electronic devices through line patterning of conducting polymers," Advanced Functional Materials, vol. 15, no. 1, pp. 51-56, 2005.

[11] G. H. Shim, M. G. Han, J. C. Sharp-Norton, S. E. Creager, and S. H. Foulger, "Inkjet-printed electrochromic devices utilizing polyaniline-silica and poly (3,4-ethylenedioxythiophene)silica colloidal composite particles," Journal of Materials Chemistry, vol. 18, no. 5, pp. 594-601, 2008.

[12] J. Kim, Y. Kim, and E. Kim, "Electrochromic pattern formation by photo cross-linking reaction of PEDOT side chains," Macromolecular Research, vol. 17, no. 10, pp. 791-796, 2009.

[13] Y. Kim, Y. Kim, S. Kim, and E. Kim, "Electrochromic diffraction from nanopatterned poly(3-hexylthiophene)," ACS Nano, vol. 4, no. 9, pp. 5277-5284, 2010.

[14] J. Kim, J. You, B. Kim, T. Park, and E. Kim, “Solution processable and patternable poly(3,4-alkylenedioxythiophene)s for large-area electrochromic films," Advanced Materials, vol. 23, no. 36, pp. 4168-4173, 2011.

[15] H. Shin, S. Seo, C. Park, J. Na, M. Han, and E. Kim, "Energy saving electrochromic windows from bistable low-HOMO level conjugated polymers," Energy \& Environmental Science, vol. 9, no. 1, pp. 117-122, 2016.

[16] S. Joo, J.-H. Kim, and S. Seo, "Direct fabrication of electrochromic devices with complex patterns on three-dimensional substrates using polymeric stencil films," RSC Advances, vol. 7, no. 68, pp. 43283-43288, 2017.

[17] H. Kim, B. Yoon, J. Sung, D.-G. Choi, and C. Park, "Micropatterning of thin $\mathrm{P} 3 \mathrm{HT}$ films via plasma enhanced polymer transfer printing," Journal of Materials Chemistry, vol. 18, no. 29, pp. 3489-3495, 2008. 
[18] H. Schmid and B. Michel, "Siloxane polymers for highresolution, high-accuracy soft lithography," Macromolecules, vol. 33, no. 8, pp. 3042-3049, 2000.

[19] J.-H. Kim, H.-S. Hwang, S.-W. Hahm, and D.-Y. Khang, "Hydrophobically recovered and contact printed siloxane oligomers for general-purpose surface patterning," Langmuir, vol. 26, no. 15, pp. 13015-13019, 2010.

[20] L. Xue and Y. Han, "Pattern formation by dewetting of polymer thin film," Progress in Polymer Science, vol. 36, no. 2, pp. 269-293, 2011.

[21] A. Kumar, D. M. Welsh, M. C. Morvant, F. Piroux, K. A. Abboud, and J. R. Reynolds, "Conducting poly (3,4-alkylenedioxythiophene) derivatives as fast electrochromics with high-contrast ratios," Chemistry of Materials, vol. 10, no. 3, pp. 896-902, 1998.

[22] S. A. Sapp, G. A. Sotzing, and J. R. Reynolds, "High contrast ratio and fast-switching dual polymer electrochromic devices," Chemistry of Materials, vol. 10, no. 8, pp. 21012108, 1998.

[23] B. D. Reeves, C. R. G. Grenier, A. A. Argun, A. Cirpan, T. D. McCarley, and J. R. Reynolds, "Spray coatable electrochromic dioxythiophene polymers with high coloration efficiencies," Macromolecules, vol. 37, no. 20, pp. 7559-7569, 2004.

[24] S. W. Huang and K. C. Ho, "An all-thiophene electrochromic device fabricated with poly (3-methylthiophene) and poly (3,4-ethylenedioxythiophene)," Solar Energy Materials \& Solar Cells, vol. 90, no. 4, pp. 491-505, 2006.

[25] Y. Pang, X. Li, H. Ding, G. Shi, and L. Jin, "Electropolymerization of high quality electrochromic poly(3-alkyl-thiophene)s via a room temperature ionic liquid," Electrochimica Acta, vol. 52, no. 20, pp. 6172-6177, 2007.

[26] J.-H. Kim, M. J. Han, and S. Seo, "Flexible, stretchable, and patchable organic devices integrated on freestanding polymeric substrates," Journal of Polymer Science, Part B: Polymer Physics, vol. 53, no. 6, pp. 453-460, 2015.

[27] K. G. Sharp, G. S. Blackman, N. J. Glassmaker, A. Jagota, and C.-Y. Hui, "Effect of stamp deformation on the quality of microcontact printing: theory and experiment," Langmuir, vol. 20, no. 15, pp. 6430-6438, 2004.

[28] S.-J. Choi, H. N. Kim, W. G. Bae, and K.-Y. Suh, "Modulusand surface energy-tunable ultraviolet-curable polyurethane acrylate: properties and applications," Journal of Materials Chemistry, vol. 21, no. 38, pp. 14325-14335, 2011.

[29] J. N. Lee, C. Park, and G. M. Whitesides, "Solvent compatibility of poly (dimethylsiloxane)-based microfluidic devices," Analytical Chemistry, vol. 75, no. 23, pp. 65446554, 2003.

[30] Z. Yoshimitsu, A. Nakajima, T. Watanabe, and K. Hashimoto, "Effects of surface structure on the hydrophobicity and sliding behavior of water droplets," Langmuir, vol. 18, no. 15, pp. 5818-5822, 2002.

[31] C. Lv, C. Yang, P. Hao, F. He, and Q. Zheng, "Sliding of water droplets on microstructured hydrophobic surfaces," Langmuir, vol. 26, no. 11, pp. 8704-8708, 2010.

[32] Y. Yoon, D.-W. Lee, and J.-B. Lee, "Surface modified nanopatterned SU-8 pillar array optically transparent superhydrophobic thin film," Journal of Micromechanics and Microengineering, vol. 22, no. 3, article 035012, 2012.

[33] C. Luo, F. Meng, and A. Francis, "Fabrication and application of silicon-reinforced PDMS masters," Microelectronics Journal, vol. 37, no. 10, pp. 1036-1046, 2006.
[34] S. Banerjee, P. H. Howard, and S. S. Lande, "General structure-vapor pressure relationships for organics," Chemosphere, vol. 21, no. 10-11, pp. 1173-1180, 1990.

[35] G. Carcano, M. Ceriani, and F. Soglio, "Spin coating with high viscosity photoresist on square substrates - applications in the thin film hybrid microwave integrated circuit field," MicroElectronics International, vol. 10, no. 3, pp. 12-20, 1993.

[36] H. Lee, K. Lee, B. Ahn, J. Xu, L. Xu, and K. W. Oh, “A new fabrication process for uniform SU-8 thick photoresist structures by simultaneously removing edge bead and air bubbles," Journal of Micromechanics and Microengineering, vol. 21, no. 12, article 125006, 2011.

[37] D. E. Motaung, G. F. Malgas, and C. J. Arendse, "Comparative study: the effects of solvent on the morphology, optical and structural features of regioregular poly(3-hexylthiophene):fullerene thin films," Synthetic Metals, vol. 160, no. 9-10, pp. 876$882,2010$. 


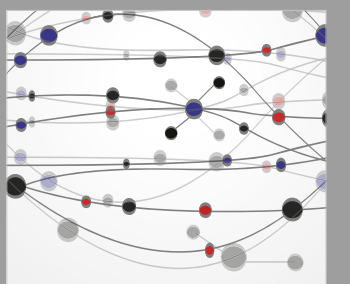

The Scientific World Journal
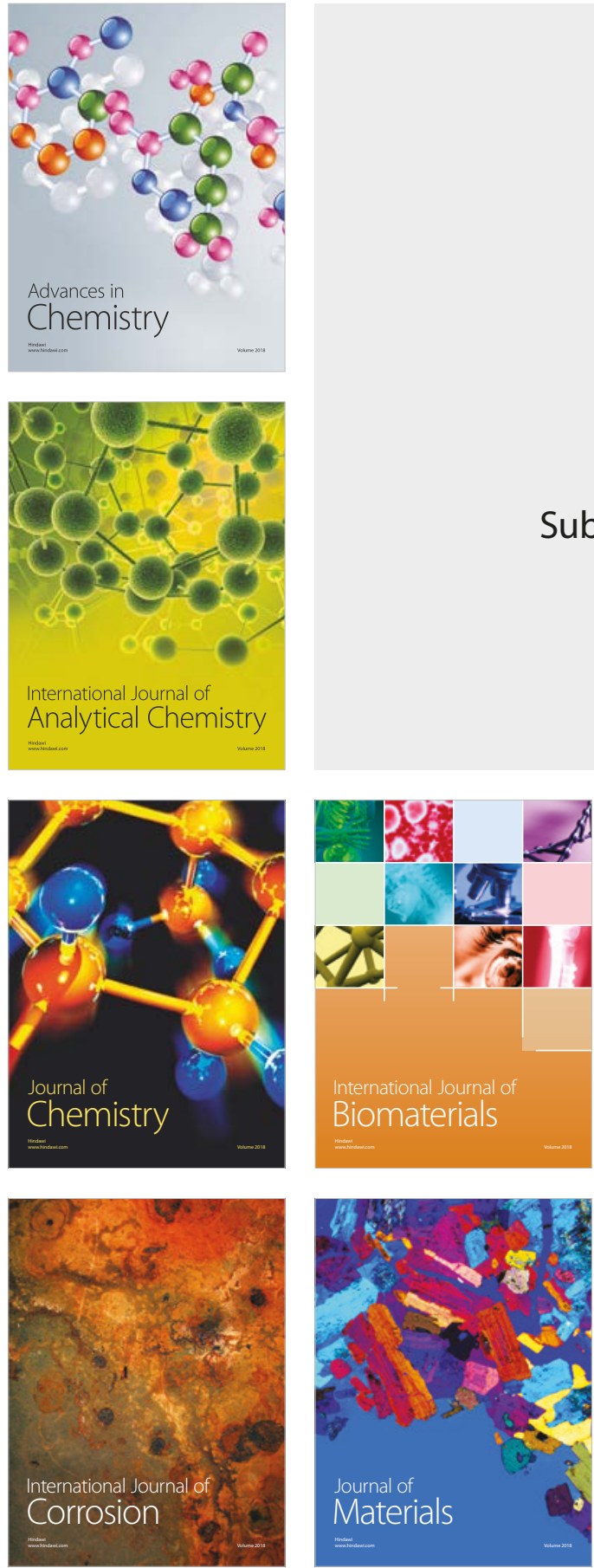

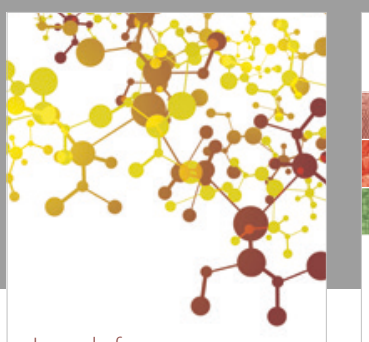

Journal of

Applied Chemistry
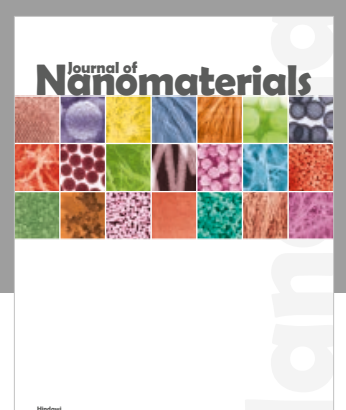

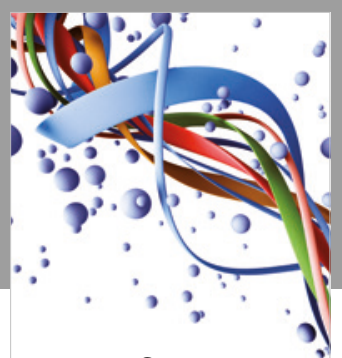

Scientifica

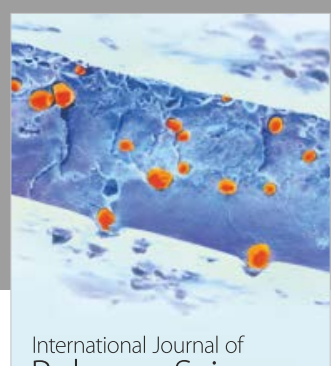

Polymer Science

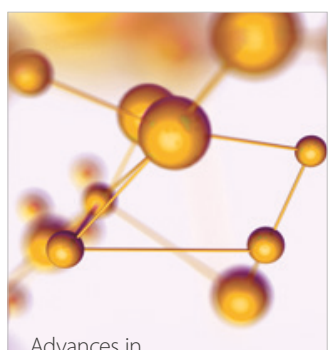

Physical Chemistry
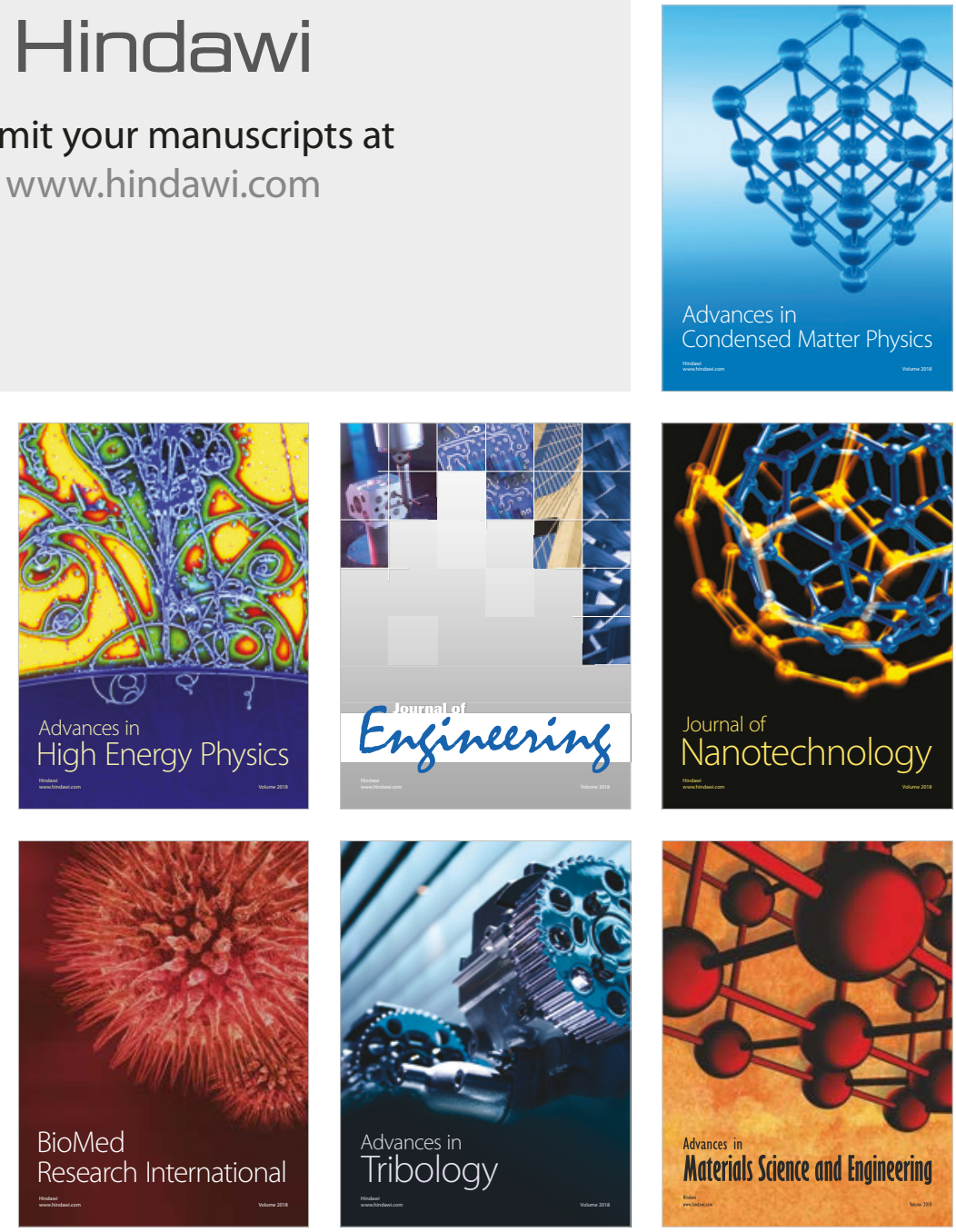